\title{
Screening of sweetpotato germplasm collections for sweetpotato weevil (Cylas spp.) resistance in Tanzania
}

\author{
Filson Kagimbo ${ }^{1,2}$, Hussein Shimelis* ${ }^{1}$, Julia Sibiya ${ }^{1}$ \\ ${ }^{1}$ African Centre for Crop Improvement, School of Agricultural, Earth and Environmental Sciences, College of \\ Agriculture, Engineering and Science, University of KwaZulu-Natal, Private Bag X01, Scottsville 3209, \\ Pietermaritzburg, South Africa \\ ${ }^{2}$ Tumbi Agricultural Research Institute, P.O. BOX 306, Tabora, Tanzania
}

\section{*Corresponding author: filisson2005@yahoo.com}

\section{Abstract}

Weevil damage caused by sweetpotato weevil (Cylas spp.) is a major constraint to sweetpotato production in Tanzania due to a lack of improved varieties with durable resistance. The objective of this study was to screen sweetpotato germplasm collections for weevil resistance and to select the best parents to be used in resistance breeding. Field studies involving 96 sweetpotato genotypes were conducted at two weevil hotspot sites in Western Tanzania using a $12 \times 8$ lattice design with three replications at each site. Data collected included yield and yield related traits, weevil reaction and weevil damage score. The tested genotypes differed significantly $(P<0.01)$ for sweetpotato storage root number, root weight, root infestation and root damage score. Weevil infestation on storage roots significantly $(P \leq 0.05)$ correlated with total root number $(r=0.38)$ and weevil damage score $(r=0.79)$. Marketable root weight and total root weight were significantly correlated with infested root weight each with $r=0.45$. The study identified nine sweetpotato genotypes expressing resistance and 10 genotypes with moderate resistance to weevil. Five genotypes including Magunhwa, Chuchu ya Nesi, Rugomoka, Tumauma and New Kawogo were selected with weevil resistance and desirable yield and yield-related traits. These genotypes can be used in future weevil resistance breeding programs of sweetpotato in Western Tanzania or related agro-ecologies.

Keywords: Resistance breeding; western Tanzania; Weevil damage; Weevil hot-spot; Storage roots.

Abbreviations: $\mathrm{ACCl}_{\text {_ }}$ African Centre for Crop Improvement; AGRA_ Alliance for a Green Revolution in Africa ; ARI_Agricultural

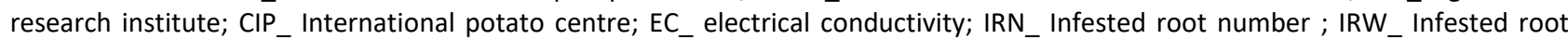
weight; LSD = Least significant difference; MAP_ Months after planting; MRN_ Markētable root number ; MRW_ Marketable root weight; OC_Organic carbon; OFSP_ Orange-fleshed sweetpotato ; Sed = Standard error of difference, ; SL_ Sandy loam,; SN= Serial number; SSA_Sub-Saharan Africa; TRN_Total root number ; TRW_Total root weight ; UN_ Unknown; WDS_Weevil damage score.

\section{Introduction}

Sweetpotato (Ipomoea batatas [L.] Lam.) is an important root crop grown in more than 110 countries worldwide on an estimated area of 8.21 million hectare (ha), with an annual production of 104.02 million tonnes (FAOSTAT, 2014). As a food crop, sweetpotato ranks seventh globally and fifth in developing countries after rice (Oryza sativa L.), wheat (Triticum aestivum L.), maize (Zea mays L.) and cassava (Manihot esculenta L.) (Elameen et al., 2008; Nelles, 2009). In sub-Saharan Africa (SSA), sweetpotato is the most important staple food crop grown on about 2.1 million ha and producing about 9.9 million tonnes fresh storage roots per annum (Anyanga et al., 2013).

In addition to food, sweetpotato is also used as a source of cash income and feed in many countries in SSA (Fuglie, 2007). The storage roots are used in various forms such as fried chips, boiled roots or as baked products (Engoru et al., 2005). The young leaves of the crop are used as leaf vegetable. In some instances the entire canopy of the crop can be used as fodder for livestock. Sweetpotato roots are rich in carbohydrates and the orange-fleshed sweetpotato varieties (OFSPs) contain high $\beta$-carotene that is a precursor of vitamin ' $A$ ' useful in combating vitamin $A$ deficiency in humans (Mwanga et al., 2007; Burri, 2011). Sweetpotato roots are also used as industrial raw material for biofuel, starch and alcohol extraction (Schafleitner et al., 2010; Clark et al., 2012).

Tanzania is the second largest producer of sweetpotato in SSA after Nigeria (FAOSTAT, 2014), where the crop is grown on an estimated area of 0.56 million ha of agricultural lands with a mean national yield of $4.55 \mathrm{t} \mathrm{ha}^{-1}$. In Tanzania, sweetpotato is cultivated in almost all agro-ecological zones under subsistence farming systems (Kulembeka et al., 2005; Masumba et al., 2005; Kagimbo et al., 2017). Sweetpotato is preferred by most rural farmers due to its high productivity per unit area, drought tolerance, early maturity and relatively good performance in marginal and poor soils 
(Kapinga et al., 1995). In most growing zones of Tanzania, farmers practice flexible planting and harvesting schedules of the crop. Further, sweetpotato fits into different cropping systems where it can be cultivated as a monocrop or intercropped with cassava, maize, beans, cowpea or groundnut (Ngailo et al., 2016a).

Sweetpotato production in Tanzania is faced with a range of constraints including biotic, abiotic and socio-economic factors which have contributed to the existing low yields of $4.62 \mathrm{t} \mathrm{ha}^{-1}$ compared to the potential productivity of the crop varying from 15 to $23 \mathrm{t} \mathrm{ha}^{-1}$ (Sebastiani et al., 2007). Amongst these constraints, sweetpotato weevil infestation caused by sweetpotato weevil (Cylas spp.) poses a major threat to sweetpotato production in Tanzania. To control the weevils, agronomic practices such as field sanitation, early planting and early harvesting, and chemical treatment have been used by farmers. However, chemical control is too expensive and unaffordable to farmers and it is less effective because the juvenile weevils develop in roots and vines (Lebot and Bradshaw, 2010). Cultural practices such as early planting are a difficult management practice for many farmers due to the shortage of planting materials at the onset of rainfall. Early harvesting is also a challenge since farmers practice sequential and piecemeal harvesting to minimise post-harvest losses. In SSA sweetpotato growers experience a range of challenges from a lack of; welldesigned storage facilities, postharvest handling facilities during packaging and transport, knowledge on processing, processing equipment and a problem of transporting bulky products (Masumba et al., 2005). Therefore, field maintained storage roots of the crop are vulnerable to several pests and diseases. Among these, sweetpotato weevils are reported to be serious insect pests damaging the crop in the field.

Breeding sweetpotato varieties with durable resistance to weevils is advocated as the best strategy to control weevils (Muyinza et al., 2012; Anyanga et al., 2013). However, weevil resistance varieties are yet to be developed and released in Tanzania. It is thus important to identify weevil resistant germplasm through effective screening involving adequate weevil infestation among trials, locations and seasons (Stathers et al., 2003). A study conducted in Tanzania and Uganda by Stathers et al. (2003) reported the existence of sweetpotato varieties exhibiting different levels of resistance to weevils, which was partly attributed to escape mechanism (Stathers et al., 2003; Muyinza et al., 2012).

In determining the level of resistance of sweetpotato varieties to weevils, test genotypes need to be subjected to the required level of pest pressure. This will ensure high levels of infestation to screen for resistance. Researchers practice artificial inoculation of laboratory reared weevils for field inoculation with a small population of the weevils for effective infestation and screening (Stathers et al., 2003; Muyinza et al., 2012). In weevil hotspot areas including western Tanzania where large population of natural weevil infestation occurs, artificial inoculation of the pest is not required (Stathers et al., 2003). The activity of artificially inoculated weevils is reported to be highly affected by environmental factors such as rainfall, and excessively low or high temperature conditions (Stathers et al., 2003).
Recently, the existence of sweetpotato varieties with reasonable levels of resistance to weevils has been reported. For instance the following varieties: New Kawogo, Dimbuka, Anamoyoto and Kyebagambire have been identified and reported to express resistance to Cylas spp. with active chemical based resistance mechanism (Stevenson et al., 2009; Muyinza et al., 2012; Anyanga et al., 2013). This type of resistance is attributed to higher levels of esters of hydroxylcinnamic acid in root latex (Stevenson et al., 2009) and esters of caffeic and coumaric acid in epidermal and root surface of resistant varieties (Anyanga et al., 2013). These chemical compounds conferring resistance to Cylas spp. are reported to be toxic to juvenile Cylas spp. but also repellent to adult Cylas spp. This indicates that more resistant varieties can possibly be selected among the local landraces. To date sweetpotato germplasm collections in Tanzania have not been effectively screened for weevil resistance breeding. Therefore, the objective of this study was to screen sweetpotato germplasm collections for sweetpotato weevil (Cylas spp) resistance in Tanzania and to select best parents to be used in breeding for weevil resistance.

\section{Results}

\section{Storage root number, root weight and weevil damage} score

There was a significant difference $(P<0.001)$ among genotypes on all the studied traits (Table 2). Location had significant effect on MRN, MRW, IRN and WDS.

Mean response of genotypes for storage roots: root number, root weight, weevil infestation and damage

The mean TRN, TRW, IRN and WDS differed significantly among the tested sweetpotato genotypes (Table 3). MRN per plot ranged from 1.69 to 19.52 with a mean of 12.12 (Table 3). The following genotypes: Kiti cha Nyerere Shitoli, Tumauma and New Kawogo had the highest MRN per plot. TRN per plot ranged from 3.20 to 36.15 with a mean of 21.06 (Table 3). Genotypes Mugandi, 7-CIP, Ukimwi and Ngw'anakasenga had the highest TRN per plot of 36.15, $34.07,33.10$ and 32.37 , in a decreasing order.

MRW per plot ranged from $0.15 \mathrm{~kg}$ to $6.77 \mathrm{~kg}$ with a mean of 3.73kg. Genotypes Ngw'ananzugi, Kiti cha Nyerere, Magunhwa and Shitoli had the highest MRW per plot of $6.77 \mathrm{~kg}, 6.55 \mathrm{~kg}, 5.87 \mathrm{~kg}$ and $5.70 \mathrm{~kg}$, in that order. The TRW per plot ranged from $0.28 \mathrm{~kg}$ to $7.40 \mathrm{~kg}$ with a mean of $4.31 \mathrm{~kg}$ (Table 3). Genotypes Ngw'ananzugi, Kiti cha Nyerere, Ukimwi and Shitoli had the highest TRW per plot of $7.40 \mathrm{~kg}$, $6.93 \mathrm{~kg}, 6.43 \mathrm{~kg}$ and $6.28 \mathrm{~kg}$, respectively.

IRW per plot ranged from $1.85 \%$ to $71.97 \%$ with a mean of $34.74 \%$. Genotypes Madebe, Kibandule, Malulumba and Utitiri had the lowest IRW per plot of $1.85 \%, 4.14 \%, 6.27 \%$ and $7.33 \%$, in that order. IRN per plot ranged from $2.01 \%$ to $59.84 \%$ with a mean of 27.84 (Table 3). Genotypes Malulumba, Kibandule, 4-CIP and Madebe had the lowest IRN of $2.01 \%, 2.40 \%, 4.87 \%$ and $5.59 \%$, in that order.

WDS ranged from $3.42 \%$ to $62.76 \%$ with a mean of $32.12 \%$ (Table 3). Genotypes with WDS between 0\% and $14.9 \%$ were categorised as resistant to the weevil. Therefore, the 
following genotypes were selected for weevil resistance: Kibandule, Malulumba, Utitiri, 3-CIP, Madebe, Magunhwa, 5-CIP, Kafu and Chuchu ya nesi. Genotypes with WDS between $15 \%$ and $20 \%$ were categorized as moderately resistant. This group included: Nyamvuva, Sengi, 22-CIP, Rugomoka, Tumauma, Ejumla, Carot C, New Kawogo 2, Haraka and 4-CIP. Genotypes with WDS between $20.1 \%$ and 24.9 were categorised as moderately susceptible. This group included Chuga, Ukerewe, UN 6, New Kawogo, 25-CIP, Kimburu, Nyaisome, Masinia M.W.N and Mulozi. Genotypes with WDS $>25 \%$ were categorized as susceptible. In general, the Ndorobo Village site had the higher percentage of MRN, IRN and WDS. ARI site had higher values of MRW. Some of the tested genotypes categorised as resistant or moderately resistant lacked good agronomic traits such as yield and yield-related traits (Table 3). Only five genotypes with weevil resistance and desirable yield were identified with data summary presented in Table 4. These genotypes included Magunhwa, Chuchu ya nesi, Rugomoka, Tumauma and New kawogo.

\section{Correlation between root number, root weight and weevil} infestation

Table 5 presents the degree of association of storage root number and root weight with root infestation and damage by weevils. Results showed that TRN was significantly $(\mathrm{P}<$ $0.05)$ correlated with IRN $(r=0.38)$. MRW and TRW were significantly $(P<0.05)$ correlated with IRW $(r=0.45)$. Further, significant correlations $(P<0.01)$ were recorded between IRN and IRW with WDS with correlation values of $r$ $=0.79$ and 0.72 , respectively (Table 5).

\section{Principal component analysis (PCA)}

Table 6 presents the results of the principal component analysis. The first three principal components described $92.66 \%$ of the total variations present among sweetpotato genotypes. Principal component 1 (PC1) was positively correlated with marketable root number $(0.42)$ and total root weight (0.42). PC2 was positively correlated with marketable root weight $(0.35)$ and total root weight $(0.34)$ but negatively correlated with weevil damage score $(-0.54)$ and infested root number $(-0.47)$. PC3 was highly and positively correlated (0.76) with total root number but negatively correlated with marketable root weight $(-0.43)$.

\section{Discussion}

\section{Storage root number and root yield}

The tested genotypes showed significant differences in total storage root number per plot (Table 3). This result is attributed to genotypic variation. Storage root formation is controlled by multiple independent genes which differ among sweetpotato varieties (Rukundo et al., 2013). The variation might also have been caused by the difference in their ability to translocate photosynthetic material from leaves to the roots. Genotypes with higher translocation ability produce more roots per unit area. Significant difference in root production among sweetpotato genotypes were reported in earlier studies (Tairo et al., 2008; Ngailo et al., 2016a).

There were significant differences in root yield per plot among the tested sweetpotato genotypes across tested locations. Genotypes with good adaptation to the test environments yield better (Masumba et al., 2005; Rukundo et al., 2013; Kagimbo et al,. 2017). Differences in maturity time are another attribute for variable root yield in sweetpotato genotypes (Lebot, 2009). Dry matter accumulation leads to high storage yield which is associated with long maturity.

Higher MRN, MRW were recorded at Ndorobo village site (Table 3). This observation is attributed to the high fertility status of the soils at Ndorobo village (Table 1 ). The soils at Ndorobo village had the higher levels of total nitrogen and organic carbon which might have favoured good growth and yield at that location. It is not surprising that even higher levels of IRN and WDS were also recorded at Ndorobo village site (Table 3). This is due to the fact that increased storage root size and root number per plot were recorded at the site that led to soil cracks exposing storage roots to weevils hence increased infestation and damage. Stathers et al. (2003) reported that increased sweetpotato yield is associated with high level of weevil infestation.

The levels of weevil infestation recorded across the two testing locations were lower but relatively the same (Table 3 ). This was related to the challenges in controlling the weevils in field. The level of weevil infestation on roots increases with time when roots remain in the field after maturity. Before sweetpotato matures, weevils (Cylas spp.) in their adult stage feed on the epidermis of vines and leaves, while larvae tunnel into the vines. When the roots enlarge and mature, they get exposed to weevil damage. At this stage the adult weevils can also feed on the surface of the roots, while larvae tunnel inside the storage roots (Skoglund and Smit, 1994). Multiplication of weevils is four times higher in storage roots than in vines (Smit et al., 2001). This implies that more weevil pressure is built after storage root maturity. Likewise, weevil infestation increases after storage root maturity.

\section{Genotypes response to weevil infestation and damage}

The present study revealed that the tested sweetpotato genotypes were significantly different regarding IRN and WDS (Table 3). Differences in the genetic constitution, environmental conditions and storage root morphology could attribute to the observed differences in response to sweetpotato weevil infestation and damage (Stathers et al., 2003; Muyinza et al., 2012). Deep root systems of some genotypes increased the distance to which weevils have to burrow to reach the roots hence reducing the weevil infestation and damage (Stathers et al., 2003). High foliage production by some genotypes protects soils from being exposed to intense solar radiation keeping moisture in the soil. This was found to reduce soil cracks and hence limit weevil infestation and damage. Production of thicker storage roots by some genotypes cause soil cracks and exposure to weevil damage (Talekar, 1987; Stathers et al., 2003). Differential response of sweetpotato varieties to weevil damage is also attributed to variation in chemical composition of storage roots (Stevenson et al., 2009; 
Table 1. Physio-chemical characteristics of soils of the study sites.

\begin{tabular}{|c|c|c|c|c|c|c|c|c|c|c|c|c|}
\hline \multirow[b]{3}{*}{ Study site } & \multicolumn{12}{|c|}{ Soil characteristics } \\
\hline & \multirow[t]{2}{*}{$\mathrm{pH}$} & \multirow[t]{2}{*}{$\begin{array}{l}\text { Total N } \\
(\%)\end{array}$} & \multirow[t]{2}{*}{ OC (\%) } & \multirow[t]{2}{*}{$\begin{array}{l}\text { Available P } \\
\text { (ppm) }\end{array}$} & \multicolumn{2}{|c|}{$\begin{array}{l}\text { Exchangeable } \\
\text { (meq/100g) }\end{array}$} & \multirow[t]{2}{*}{ bases } & \multirow[t]{2}{*}{$\begin{array}{l}\mathrm{EC} \\
(\mathrm{mS} / \mathrm{cm})\end{array}$} & \multicolumn{3}{|c|}{ Texture (\%) } & \multirow[t]{2}{*}{$\begin{array}{l}\text { Textural } \\
\text { class }\end{array}$} \\
\hline & & & & & $\mathrm{Mg}$ & $\mathrm{Ca}$ & & & Sand & Silt & Clay & \\
\hline Ndorobo & 6.2 & 0.13 & 0.63 & 17.5 & 1.22 & 0.22 & & 0.06 & 71 & 9 & 20 & $S L$ \\
\hline ARI-Tumbi & 6.1 & 0.04 & 0.270 & 16.1 & 0.04 & 0.23 & & 1.62 & 75 & 9 & 16 & SL \\
\hline
\end{tabular}

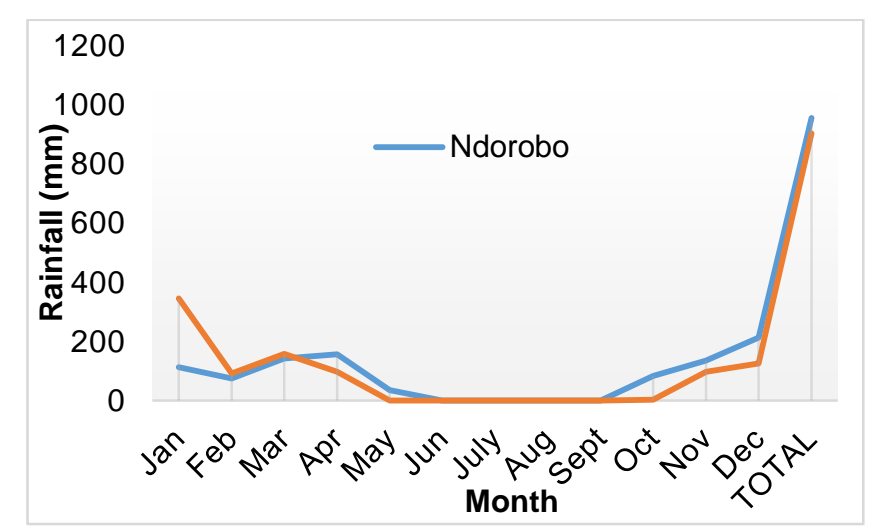

Fig 1. Rainfall distribution at the sites during the study season.

Table 2. Restricted maximum likelihood analysis of root number, root weight and root damage score of 96 sweetpotato genotypes evaluated across two sites.

\begin{tabular}{|c|c|c|c|c|c|c|c|c|}
\hline \multirow{2}{*}{$\begin{array}{l}\text { Source } \\
\text { variation }\end{array}$} & \multirow[t]{2}{*}{ of } & \multicolumn{7}{|c|}{ Wald statistic } \\
\hline & & MRN & TRN & MRW & TRW & IRN & IRW & WDS \\
\hline Replication & 2 & & & & & & & \\
\hline Genotype & 95 & $272.04 * * *$ & $275.01 * * *$ & $197.94 * * *$ & $192.23 * * *$ & $156.54 * * *$ & $141.94^{* * *}$ & $134.75^{* *}$ \\
\hline Location & 1 & $15.81 * * *$ & 0.19 & $7.29 * *$ & 1.75 & $5.67 *$ & 3.05 & $4.35^{*}$ \\
\hline Genotype & $*$ & & & & & & & \\
\hline Location & 95 & 74.22 & 54.51 & 54.35 & 57.83 & 42.94 & 41.43 & 32.41 \\
\hline
\end{tabular}

Error $\quad 192$
$\mathrm{DF}=\frac{\text { degree of freedom, }}{* * *}=$ significant at $\mathrm{P} \leq 0.001,{ }^{* *}=$ significant at $\mathrm{P} \leq 0.01,{ }^{*}$ significant at $\mathrm{P}<0.05, \mathrm{MRN}=$ Marketable root number per plot, TRN $=$ Total root number per plot, $\mathrm{MRW}=$
Marketable root weight per plot $(\mathrm{kg} / \mathrm{plot}), \mathrm{TRW}=$ Total root weight per lot $(\mathrm{kg} / \mathrm{plot}), \mathrm{IRN}=$ Infested root number per plot, IRW = Infested root weight per plot $(\mathrm{kg} / \mathrm{plot})$, WDS $=$ Weevil damage score.

Table 3. Mean values of seven traits of 96 sweetpotato genotypes evaluated at two sites in western Tanzania.

\begin{tabular}{|c|c|c|c|c|c|c|c|c|}
\hline $\mathrm{SN}$ & Genotype & MRN & TRN & MRW & TRW & IRN & IRW & WDS \\
\hline 1 & Komando & $12.86^{e-y}$ & $21.70^{f-A}$ & $5.099^{u-c}$ & $5.892^{y-1}$ & $8.573^{g-v}$ & $3.395^{r-x}$ & $45.25^{\mathrm{m}-\mathrm{z}}$ \\
\hline 2 & Vitaa K & $14.69^{p-E}$ & $30.44^{A-G}$ & $4.334^{\mathrm{I}-\mathrm{A}}$ & $5.026^{1-H}$ & $7.837^{\mathrm{e}-\mathrm{u}}$ & $2.028^{d-w}$ & $30.06^{b-w}$ \\
\hline 3 & Mvumbagu & $11.38^{b-u}$ & $14.72^{b-k}$ & $3.870^{f-z}$ & $4.181^{C-B}$ & $7.486^{d-t}$ & $2.796^{0-x}$ & $42.74^{k-2}$ \\
\hline 4 & Kimburu & $16.05 t^{-E}$ & $25.05^{\mathrm{m}-\mathrm{F}}$ & $5.311^{w-c}$ & $5.977^{z-1}$ & $5.68^{a-s}$ & $1.728^{b-r}$ & $23.44^{a-q}$ \\
\hline 5 & Ntegakatebo & $12.74^{e-y}$ & $21.73^{\mathrm{g}-\mathrm{A}}$ & $3.503^{b-x}$ & $4.205^{C-D}$ & $8.325^{\mathrm{g}-\mathrm{v}}$ & $1.191^{\mathrm{a}-\mathrm{o}}$ & $28.05^{a-u}$ \\
\hline 6 & 8-CIP & $12.65^{d-y}$ & $21.47^{f-A}$ & $4.623^{\mathrm{q}-\mathrm{A}}$ & $5.279^{\mathrm{O}-\mathrm{H}}$ & $6.322^{\mathrm{a}-\mathrm{s}}$ & $2.317^{h-x}$ & $36.92^{\mathrm{d}-\mathrm{z}}$ \\
\hline 7 & 18-CIP & $15.92^{\text {S-E }}$ & $28.32^{W-G}$ & $4.281^{\mathrm{k}-\mathrm{A}}$ & $4.985^{1-H}$ & $10.049^{1-x}$ & $2.642^{1-x}$ & $44.16^{\mathrm{m}-\mathrm{z}}$ \\
\hline 8 & Ukerewe & $8.40^{b-h}$ & $10.89^{a-c}$ & $3.598^{c-x}$ & $3.888^{b-x}$ & $4.59^{a-o}$ & $1.015^{\mathrm{a}-\mathrm{l}}$ & $20.47^{a-n}$ \\
\hline 9 & Ngw'anangusa & $9.57^{b-n}$ & $19.28^{c-w}$ & $2.625^{b-o}$ & $3.407^{b-p}$ & $3.533^{a-j}$ & $0.681^{a-h}$ & $25.81^{a-t}$ \\
\hline 10 & $17-\mathrm{CIP}$ & $7.10^{b-c}$ & $15.10^{b-k}$ & $1.620^{a-b}$ & $2.001^{a-b}$ & $3.707^{a-1}$ & $0.913^{a-j}$ & $31.30^{c-x}$ \\
\hline 11 & Kalamu & $10.76^{b-r}$ & $17.34^{c-o}$ & $4.237^{\mathrm{j}-\mathrm{A}}$ & $4.625^{f-G}$ & $4.84^{a-q}$ & $1.707^{b-q}$ & $25.78^{a-s}$ \\
\hline 12 & 13-CIP & $7.70^{b-d}$ & $10.66^{a-c}$ & $2.364^{b-k}$ & $2.503^{b-d}$ & $5 .^{512 \mathrm{a}-\mathrm{r}}$ & $1.767^{c-s}$ & $29.67^{a-w}$ \\
\hline 13 & 25-CIP & $9.70^{b-p}$ & $19.50^{c-w}$ & $2.159^{b-g}$ & $2.605^{b-e}$ & $3.30^{a-i}$ & $0.837^{a-i}$ & $23.27^{\mathrm{a}-\mathrm{q}}$ \\
\hline 14 & Chuchu ya nesi & $15.70^{r-E}$ & $31.70^{C-G}$ & $4.984^{s-c}$ & $5.759^{u-1}$ & $2.753^{a-g}$ & $0.982^{a-1}$ & $14.32^{a-h}$ \\
\hline 15 & Magunhwa & $12.76^{e-y}$ & $17.94^{c-t}$ & $5.868^{A-C}$ & $6.282^{\mathrm{F}-\mathrm{I}}$ & $3.613^{a-k}$ & $1.506^{a-p}$ & $12.80^{\mathrm{a}-\mathrm{e}}$ \\
\hline 16 & Sengi & $12.63^{d-y}$ & $17.47^{c-p}$ & $4.033^{g-A}$ & $4.566^{\mathrm{e}-\mathrm{G}}$ & $1.229^{\mathrm{a}-\mathrm{d}}$ & $0.332^{a-c}$ & $15.31^{a-i}$ \\
\hline 17 & Jewel & $9.65^{b-o}$ & $31.31^{C-G}$ & $1.871^{a-d}$ & $2.650^{b-f}$ & $11.331^{\mathrm{rx}}$ & $1.199^{a-o}$ & $51.06^{r-z}$ \\
\hline 18 & Ejumla & $10.69^{b-q}$ & $21.77^{\mathrm{g}-\mathrm{A}}$ & $3.099^{b-t}$ & $3.751^{b-u}$ & $2.353^{\mathrm{a}-\mathrm{g}}$ & $0.822^{a-i}$ & $18.99^{a-m}$ \\
\hline 19 & Masinia M.W.N & $10.18^{b-p}$ & $14.01^{b-i}$ & $3.611^{c-x}$ & $3.800^{b-u}$ & $5.27^{\mathrm{a}-\mathrm{r}}$ & $1.655^{a-q}$ & $24.24^{a-q}$ \\
\hline 20 & UN 2 & $13.70^{\mathrm{j}-\mathrm{B}}$ & $24.54^{\text {I-E }}$ & $4.561^{p-A}$ & $5.244^{n-H}$ & $14.302^{v-x}$ & $3.424^{5-x}$ & $48.26^{p-z}$ \\
\hline 21 & UN 6 & $13.14^{\mathrm{g}-\mathrm{A}}$ & $21.19^{\mathrm{e}-\mathrm{z}}$ & $4.976^{t-c}$ & $5.617^{t-1}$ & $3.40^{a-i}$ & $0.940^{a-k}$ & $21.47^{\mathrm{a}-\mathrm{o}}$ \\
\hline 22 & Rugomoka & $18.04^{A-E}$ & $28.78^{x-G}$ & $5.118^{u-c}$ & $5.863^{v-1}$ & $3.30^{a-i}$ & $0.974^{a-1}$ & $16.28^{a-k}$ \\
\hline 23 & $5-\mathrm{CIP}$ & $7.66^{\mathrm{b}-\mathrm{d}}$ & $15.15^{b-k}$ & $3.012^{b-r}$ & $3.567^{\mathrm{b}-\mathrm{s}}$ & $2.73^{a-g}$ & $1.113^{a-n}$ & $13.50^{a-f}$ \\
\hline 24 & Kafu & $12.38^{d-y}$ & $19.34^{c-w}$ & $3.962^{f-A}$ & $4.572^{\mathrm{e}-\mathrm{G}}$ & $1.49^{\mathrm{a}-\mathrm{e}}$ & $0.685^{a-h}$ & $13.65^{a-g}$ \\
\hline 25 & Awilo & $12.00^{c-x}$ & $22.75^{\mathrm{i}-\mathrm{C}}$ & $3.588^{c-x}$ & $4.128^{c-A}$ & $8.000 f^{v}$ & $2.065^{e-w}$ & $40.51^{h-z}$ \\
\hline 26 & Kabelele & $9.14^{b-1}$ & $15.41^{b-1}$ & $2.353^{b-j}$ & $3.728^{b-t}$ & $6.461^{a-s}$ & $1.817^{c-v}$ & $41.53^{i-z}$ \\
\hline 27 & Kasinia & $14.24^{m-D}$ & $21.78^{\mathrm{g}-\mathrm{A}}$ & $4.210^{\mathrm{j}-\mathrm{A}}$ & $4.765^{h-G}$ & $10.753^{p-x}$ & $2.910^{p-x}$ & $54.41^{\mathrm{u}-\mathrm{z}}$ \\
\hline
\end{tabular}




\begin{tabular}{|c|c|c|c|c|c|c|c|c|}
\hline 28 & Kakamega & $11.28^{\mathrm{b}-\mathrm{u}}$ & $18.85^{\mathrm{c}-\mathrm{v}}$ & $4.137^{i-A}$ & $4.823^{j-G}$ & $5.421^{\mathrm{a}-\mathrm{r}}$ & $1.597^{\mathrm{a}-\mathrm{q}}$ & $35.41^{d-y}$ \\
\hline 29 & $10-\mathrm{CIP}$ & $11.55^{b-v}$ & $27.31^{\mathrm{u}-\mathrm{G}}$ & $2.355^{b-j}$ & $3.360^{b-p}$ & $7.097^{c-s}$ & $1.106^{a-m}$ & $31.12^{b-x}$ \\
\hline 30 & Ukimwi & $16.39^{\mathrm{V}-\mathrm{E}}$ & $33.10^{\mathrm{E}-\mathrm{G}}$ & $5.186^{v-C}$ & $6.430^{\mathrm{G}-1}$ & $7.125^{c-s}$ & $1.972^{c-w}$ & $28.41^{a-v}$ \\
\hline 31 & Nkima ati na siri & $15.34^{\mathrm{q}-\mathrm{E}}$ & $23.46^{\mathrm{j}-\mathrm{D}}$ & $5.101^{u-c}$ & $5.614^{t-1}$ & $4.233^{\mathrm{a}-\mathrm{m}}$ & $1.177^{\mathrm{a}-\mathrm{o}}$ & $27.80^{a-t}$ \\
\hline 32 & Malulumba & $12.98^{f-z}$ & $22.20^{h-B}$ & $3.501^{b-x}$ & $4.154^{c-A}$ & $0.541^{a}$ & $0.326^{a-c}$ & $04.68^{a-b}$ \\
\hline 33 & Masinia njano & $11.91^{c-x}$ & $21.79^{\mathrm{g}-\mathrm{A}}$ & $3.447^{b-x}$ & $4.111^{\mathrm{C}-\mathrm{A}}$ & $7.446^{d-t}$ & $1.519^{a-p}$ & $34.66^{d-y}$ \\
\hline 34 & Burenda & $7.86^{\mathrm{b}-\mathrm{e}}$ & $16.34^{b-m}$ & $2.555^{b-m}$ & $3.116^{b-1}$ & $5.731^{a-s}$ & $1.461^{a-p}$ & $28.40^{a-v}$ \\
\hline 35 & Ntulawima & $10.71^{b-r}$ & $17.45^{c-p}$ & $3.381^{b-v}$ & $3.875^{b-w}$ & $7.008^{b-s}$ & $1.553^{a-p}$ & $40.02^{f-z}$ \\
\hline 36 & Ndezu ya ntemi & $10.65^{b-q}$ & $19.43^{c-w}$ & $3.207^{\mathrm{b}-\mathrm{u}}$ & $3.823^{b-u}$ & $3.645^{a-k}$ & $1.139^{\mathrm{a}-\mathrm{o}}$ & $26.29^{a-t}$ \\
\hline 37 & Nyamvuva & $12.83^{e-y}$ & $18.23^{c-u}$ & $3.627^{c-x}$ & $3.946^{b-y}$ & $3.658^{a-k}$ & $0.974^{a-1}$ & $15.27^{a-i}$ \\
\hline 38 & Lusafisha & $13.58^{i-B}$ & $20.12^{d-x}$ & $4.533^{n-A}$ & $5.063^{1-H}$ & $2.93^{\mathrm{a}-\mathrm{g}}$ & $0.769^{a-i}$ & $26.54^{\mathrm{a}-\mathrm{t}}$ \\
\hline 39 & Vitaa U & $10.09^{b-p}$ & $18.76^{c-v}$ & $2.496^{b-1}$ & $2.987^{b-k}$ & $3.267^{a-i}$ & $0.694^{a-h}$ & $37.27^{d-z}$ \\
\hline 40 & Utitiri & $8.14^{\text {b-f }}$ & $11.56^{a-d}$ & $3.079^{b-s}$ & $3.341^{b-p}$ & $0.982^{a-c}$ & $0.445^{a-e}$ & $7.86^{a-c}$ \\
\hline 41 & Ngw'anakasenga & $12.13^{d-x}$ & $32.37^{D-G}$ & $2.434^{b-1}$ & $3.442^{b-p}$ & $9.562^{i-x}$ & $1.416^{a-p}$ & $35.54^{d-y}$ \\
\hline 42 & Haraka & $10.94^{b-s}$ & $20.22^{d-x}$ & $2.681^{b-p}$ & $4.580^{\mathrm{e}-\mathrm{G}}$ & $3.014^{a-h}$ & $0.503^{a-f}$ & $19.84^{a-m}$ \\
\hline 43 & UN 4 & $11.70^{b-v}$ & $18.62^{c-v}$ & $3.796^{\mathrm{e}-\mathrm{z}}$ & $4.449^{d-G}$ & $3.97^{a-1}$ & $1.097^{\mathrm{a}-\mathrm{m}}$ & $31.48^{c-x}$ \\
\hline 44 & China & $13.98^{k-C}$ & $19.51^{c-w}$ & $4.149^{\mathrm{i}-\mathrm{A}}$ & $4.621^{f-G}$ & $10.346^{m-x}$ & $2.713^{m-x}$ & $41.23^{i-z}$ \\
\hline 45 & Carot C & $11.10^{\mathrm{b}-\mathrm{t}}$ & $25.60^{n-F}$ & $2.060^{a-f}$ & $2.913^{b-j}$ & $2.498^{\mathrm{a}-\mathrm{g}}$ & $0.360^{a-d}$ & $19.38^{a-m}$ \\
\hline 46 & Magazi & $11.76^{b-w}$ & $21.50^{f-A}$ & $4.079^{h-A}$ & $4.676^{\mathrm{g}-\mathrm{G}}$ & $4.750^{a-p}$ & $1.731^{b-r}$ & $37.97^{d-z}$ \\
\hline 47 & Mabangili & $14.61^{\mathrm{O}-\mathrm{E}}$ & $23.33^{j-D}$ & $5.611^{y-c}$ & $6.230^{E-I}$ & $3.23^{a-i}$ & $1.197^{\mathrm{a}-\mathrm{o}}$ & $30.47^{b-w}$ \\
\hline 48 & $3-\mathrm{CIP}$ & $10.47^{b-q}$ & $21.76^{g-A}$ & $3.965^{g-A}$ & $4.593^{e-G}$ & $2.576^{a-g}$ & $0.358^{a-d}$ & $11.63^{a-d}$ \\
\hline 49 & 2-CIP & $10.45^{b-q}$ & $21.39^{f-A}$ & $2.609^{b-m}$ & $3.231^{b-n}$ & $5.413^{a-r}$ & $0.956^{a-k}$ & $28.04^{a-u}$ \\
\hline 50 & $12-\mathrm{CIP}$ & $9.02^{b-k}$ & $15.95^{b-m}$ & $2.969^{b-r}$ & $3.533^{b-r}$ & $3.235^{a-i}$ & $0.919^{a-j}$ & $25.21^{a-r}$ \\
\hline 51 & Secondary & $10.11^{b-p}$ & $13.24^{b-g}$ & $3.523^{b-x}$ & $4.075^{\mathrm{c}-\mathrm{A}}$ & $6.843^{e-s}$ & $1.771^{c-t}$ & $45.78^{p-z}$ \\
\hline 52 & Masinia nyeupe & $15.33^{q-E}$ & $26.43^{\mathrm{o-F}}$ & $4.623^{\mathrm{q}-\mathrm{A}}$ & $5.328^{p-H}$ & $6.022^{a-s}$ & $1.433^{a-p}$ & $30.90^{b-x}$ \\
\hline 53 & $11-\mathrm{CIP}$ & $11.45^{b-v}$ & $17.97^{c-t}$ & $3.769^{e-y}$ & $4.022^{c-z}$ & $5.279^{\mathrm{a}-\mathrm{r}}$ & $1.641^{a-q}$ & $32.14^{c-x}$ \\
\hline 54 & Kabode & $14.04^{I-C}$ & $21.66^{f-A}$ & $4.503^{n-A}$ & $4.905^{j-G}$ & $5.027^{\mathrm{a}-\mathrm{r}}$ & $1.351^{a-p}$ & $27.02^{a-t}$ \\
\hline 55 & 20-CIP & $9.48^{b-n}$ & $16.23^{b-m}$ & $2.292^{b-i}$ & $2.726^{\mathrm{b}-\mathrm{g}}$ & $1.699^{a-f}$ & $0.580^{\mathrm{a}-\mathrm{g}}$ & $33.01^{c-x}$ \\
\hline 56 & 4-CIP & $10.12^{b-p}$ & $13.87^{b-i}$ & $3.371^{b-v}$ & $3.726^{b-t}$ & $0.71^{a-b}$ & $0.330^{a-c}$ & $19.88^{a-m}$ \\
\hline 57 & New Kawogo & $18.32^{B-E}$ & $28.36^{w-G}$ & $4.953^{s-c}$ & $5.581^{s-1}$ & $4.220^{\mathrm{a}-\mathrm{m}}$ & $1.164^{\mathrm{a}-\mathrm{o}}$ & $21.47^{a-o}$ \\
\hline 58 & Pananzala14-CIP & $6.83^{b}$ & 12.23 a-e & $3.395^{b-w}$ & $3.835^{b-u}$ & $4.984^{\mathrm{a}-\mathrm{r}}$ & $2.247^{g-x}$ & $35.50^{d-y}$ \\
\hline 59 & $14-\mathrm{CIP}$ & $8.80^{b-j}$ & $19.48^{c-w}$ & $2.199^{b-h}$ & $2.753^{b-h}$ & $6.588^{a-s}$ & $1.555^{a-p}$ & $44.59^{\mathrm{m}-\mathrm{z}}$ \\
\hline 60 & Simama & $9.86^{b-p}$ & $17.86^{c-s}$ & $3.251^{b-u}$ & $3.737^{b-t}$ & $5.65^{a-r}$ & $1.638^{a-q}$ & $38.47^{\mathrm{e}-\mathrm{z}}$ \\
\hline 61 & Wangeni & $13.02^{f-z}$ & $25.97^{\mathrm{o}-\mathrm{F}}$ & $3.286^{b-v}$ & $3.973^{b-y}$ & $14.226^{v-x}$ & $2.790^{n-x}$ & $62.76^{2}$ \\
\hline 62 & $7-\mathrm{CIP}$ & $15.23^{\mathrm{q}-\mathrm{E}}$ & $34.07^{\mathrm{F}-\mathrm{G}}$ & $3.219^{\mathrm{b}-\mathrm{u}}$ & $4.195^{c-c}$ & $14.019^{u-x}$ & $2.063^{e-w}$ & $54.79^{v-z}$ \\
\hline 63 & Carot Dar & $15.39^{\mathrm{q}-\mathrm{E}}$ & $27.45^{v-G}$ & $4.228^{\mathrm{j}-\mathrm{A}}$ & $4.940^{\mathrm{k}-\mathrm{H}}$ & $8.415^{\mathrm{g}-\mathrm{v}}$ & $2.160^{f-w}$ & $37.37^{d-z}$ \\
\hline 64 & 24-CIP & $11.04^{b-s}$ & $29.42^{y-G}$ & $2.617^{b-n}$ & $3.293^{b-o}$ & $8.594^{h-w}$ & $2.033^{e-w}$ & $40.47^{\mathrm{m}-\mathrm{z}}$ \\
\hline 65 & Kibandule & $1.69^{a}$ & $3.20^{\mathrm{a}}$ & $0.146^{a}$ & $0.283^{a}$ & $0.41^{\mathrm{a}}$ & $0.015^{a}$ & $3.42^{\mathrm{a}}$ \\
\hline 66 & Chrolophenical & $8.24^{b-g}$ & $14.35^{b-j}$ & $2.342^{b-j}$ & $2.773^{b-i}$ & $7.049^{b-s}$ & $2.191^{\mathrm{g}-\mathrm{w}}$ & $47.63^{0-z}$ \\
\hline 67 & UN 7 & $8.18^{b-g}$ & $12.56^{b-f}$ & $3.003^{b-r}$ & $3.255^{b-n}$ & $4.237^{a-m}$ & $1.705^{b-q}$ & $37.56^{d-z}$ \\
\hline 68 & Kajiji & $16.79^{x-E}$ & $27.52^{v-G}$ & $4.941^{s-c}$ & $5.687^{t-1}$ & $13.715^{\mathrm{t}-\mathrm{x}}$ & $3.495^{v-x}$ & $47.46^{0-z}$ \\
\hline 69 & UN 3 & $11.46^{b-v}$ & $19.47^{c-w}$ & $3.625^{c-x}$ & $4.053^{C-A}$ & $5.356^{\mathrm{a}-\mathrm{r}}$ & $1.276^{\mathrm{a}-\mathrm{p}}$ & $40.13^{g-2}$ \\
\hline 70 & Ngeni & $11.90^{c-x}$ & $17.57^{c-q}$ & $3.778^{d-y}$ & $4.051^{c-z}$ & $9.886^{j-x}$ & $3.270^{q-x}$ & $49.74^{q-z}$ \\
\hline 71 & Nyaisome & $13.29^{h-A}$ & $21.92^{\mathrm{g}-\mathrm{A}}$ & $5.629^{y-c}$ & $6.180^{\mathrm{B}-1}$ & $3.844^{a-1}$ & $2.155^{f-w}$ & $23.59^{a-a}$ \\
\hline 72 & Madebe & $7.10^{b-c}$ & $7.88^{a-b}$ & $2.939^{b-r}$ & $3.054^{b-1}$ & $0.50^{\mathrm{a}}$ & $0.076^{a-b}$ & $12.31^{\mathrm{a}-\mathrm{e}}$ \\
\hline 73 & Kabakuli & $10.22^{b-p}$ & $13.36^{b-h}$ & $4.033^{g-A}$ & $4.215^{\mathrm{C}-\mathrm{E}}$ & $6.647^{a-s}$ & $2.570^{j-x}$ & $40.91^{\mathrm{i}-z}$ \\
\hline 74 & 9-CIP & $10.22^{b-q}$ & $20.78^{e-y}$ & $2.731^{b-q}$ & $3.216^{\mathrm{b}-\mathrm{m}}$ & $10.642^{n-x}$ & $2.410^{i-x}$ & $60.99^{y-z}$ \\
\hline 75 & Polista & $11.94^{c-x}$ & $19.11^{c-v}$ & $2.950^{b-r}$ & $3.593^{b-s}$ & $5.805^{a-s}$ & $1.343^{a-p}$ & $40.13^{g-z}$ \\
\hline 76 & Shitoli & $19.20^{\mathrm{D}-\mathrm{E}}$ & $29.99^{z-G}$ & $5.704^{z-C}$ & $6.283^{F-1}$ & $14.004^{u-x}$ & $3.632^{w-x}$ & $42.20^{\mathrm{j}-z}$ \\
\hline 77 & Ngw'alu & $17.22^{y-E}$ & $27.38^{v-G}$ & $4.784^{r-B}$ & $5.510^{r-1}$ & $9.903^{k-x}$ & $2.758^{m-x}$ & $55.49^{w-z}$ \\
\hline 78 & $6-\mathrm{CIP}$ & $16.39^{\text {W-E }}$ & $31.09^{B-G}$ & $4.836^{r-B}$ & $5.483^{q-1}$ & $6.700^{a-s}$ & $2.598^{k-x}$ & $42.50^{\mathrm{k}-\mathrm{z}}$ \\
\hline 79 & Tumauma & $18.74^{C-E}$ & $26.24^{\mathrm{o}-\mathrm{F}}$ & $5.617^{y-c}$ & $6.046^{A-1}$ & $2.341^{\mathrm{a}-\mathrm{g}}$ & $0.785^{a-i}$ & $16.84^{a-1}$ \\
\hline 80 & Kisu cha Masai & $14.35^{M-D}$ & $24.99^{\mathrm{m}-\mathrm{F}}$ & $4.755^{r-B}$ & $5.291^{\mathrm{O}-\mathrm{H}}$ & $6.560^{a-s}$ & $1.541^{a-p}$ & $34.08^{c-x}$ \\
\hline 81 & $22-\mathrm{CIP}$ & $8.33^{b-h}$ & $26.50^{p-F}$ & $1.779^{a-c}$ & $2.651^{b-f}$ & $2.450^{\mathrm{a}-\mathrm{g}}$ & $0.441^{a-e}$ & $15.83^{a-j}$ \\
\hline 82 & Ndovadoe & $9.39^{b-m}$ & $17.60^{c-r}$ & $1.930^{\mathrm{a}-\mathrm{e}}$ & $2.358^{b-c}$ & $5.236^{a-r}$ & $0.844^{a-i}$ & $29.35^{a-w}$ \\
\hline 83 & Ngw'anakurwa & $8.62^{b-i}$ & $12.11^{\mathrm{a}-\mathrm{e}}$ & $3.114^{\mathrm{b}-\mathrm{t}}$ & $3.477^{b-q}$ & $4.67^{a-p}$ & $1.634^{\mathrm{a}-\mathrm{q}}$ & $43.01^{1-z}$ \\
\hline 84 & Mwanatata & $13.41^{\mathrm{i}-\mathrm{B}}$ & $23.52^{k-D}$ & $4.441^{\mathrm{m}-\mathrm{A}}$ & $5.136^{\mathrm{m}-\mathrm{H}}$ & $4.833^{a-p}$ & $1.741^{b-r}$ & $29.25^{a-w}$ \\
\hline 85 & Mulozi & $13.91^{k-c}$ & $26.41^{\mathrm{o-F}}$ & $4.164^{\mathrm{i}-\mathrm{A}}$ & $4.775^{i-G}$ & $5.137^{\mathrm{a}-\mathrm{r}}$ & $1.492^{a-p}$ & $24.91^{a-r}$ \\
\hline 86 & Ngw'ananzugi & $17.98^{z-E}$ & $26.72^{q-F}$ & $6.771^{c}$ & $7.395^{\prime}$ & $6.430^{a-s}$ & $2.158^{f-w}$ & $33.64^{c-x}$ \\
\hline 87 & Chuga & $11.59^{b-v}$ & $13.72^{b-i}$ & $3.977^{\mathrm{g}-\mathrm{A}}$ & $4.132^{c-A}$ & $2.96^{\mathrm{a}-\mathrm{g}}$ & $1.228^{\mathrm{a}-\mathrm{o}}$ & $20.19^{a-m}$ \\
\hline 88 & Naspot 1 & $9.91^{b-p}$ & $15.94^{b-m}$ & $3.406^{b-x}$ & $3.852^{b-v}$ & $9.856^{j-x}$ & $1.855^{c-v}$ & $34.98^{d-y}$ \\
\hline 89 & Kiti cha Nyerere & $19.52^{\mathrm{E}}$ & $27.52^{v-G}$ & $6.552^{B-C}$ & $6.934^{\mathrm{H}-\mathrm{I}}$ & $14.082^{\mathrm{u}-\mathrm{x}}$ & $3.894^{x}$ & $54.79^{v-z}$ \\
\hline 90 & UN 1 & $13.10^{f-A}$ & $21.41^{\mathrm{f}-\mathrm{A}}$ & $4.131^{\mathrm{i}-\mathrm{A}}$ & $4.544^{\mathrm{e}-\mathrm{G}}$ & $7.322^{c-s}$ & $1.489^{a-p}$ & $38.48^{e-z}$ \\
\hline 91 & New Dimbuka & $10.58^{b-q}$ & $17.34^{c-0}$ & $3.753^{d-y}$ & $4.272^{c-F}$ & $6.755^{a-s}$ & $1.720^{b-r}$ & $43.32^{1-z}$ \\
\hline 92 & SPKBH/03/03 & $14.34^{\mathrm{m}-\mathrm{D}}$ & $17.78^{c-s}$ & $5.301^{x-C}$ & $5.540^{r-1}$ & $4.355^{a-n}$ & $1.758^{c-s}$ & $27.46^{\mathrm{a}-\mathrm{t}}$ \\
\hline 93 & $21-\mathrm{CIP}$ & $15.42^{q-E}$ & $27.05^{t-G}$ & $3.748^{d-y}$ & $4.388^{d-F}$ & $8.313^{g-v}$ & $1.808^{c-u}$ & $30.20^{b-w}$ \\
\hline 94 & Mugandi & $16.28^{\mathrm{u}-\mathrm{E}}$ & $36.15^{\mathrm{G}}$ & $3.989^{g-A}$ & $5.287^{\mathrm{O}-\mathrm{H}}$ & $10.748^{p-x}$ & $2.124^{f-w}$ & $44.25^{\mathrm{m}-\mathrm{z}}$ \\
\hline 95 & Uchungu wa mbwa & $14.43^{n-D}$ & $24.81^{\mathrm{m}-\mathrm{E}}$ & $3.201^{b-u}$ & $3.786^{b-u}$ & $12.045^{s-x}$ & $2.446^{i-x}$ & $57.46^{x-z}$ \\
\hline
\end{tabular}




\begin{tabular}{|c|c|c|c|c|c|c|c|c|}
\hline 96 & New Kawogo 2 & $9.19^{b-1}$ & $17.50^{c-p}$ & $3.218^{b-u}$ & $3.800^{b-u}$ & $4.233^{a-m}$ & $1.427^{a-p}$ & $1.979^{\mathrm{a}-\mathrm{m}}$ \\
\hline & Grand mean & 12.12 & 21.06 & 3.733 & 4.314 & 5.977 & 1.584 & 3.212 \\
\hline & LSD (5\%) & 5.005 & 9.152 & 1.918 & 2.017 & 6.360 & 1.668 & 2.657 \\
\hline & Sed & 2.542 & 4.635 & 0.9726 & 1.022 & 3.201 & 0.8515 & 1.337 \\
\hline & \multicolumn{8}{|l|}{ Location Means } \\
\hline & ARI Tumbi & 37.61 & 21.21 & 88.65 & 4.407 & 25.61 & 33.47 & 30.00 \\
\hline & Ndorobo & 47.31 & 20.91 & 82.50 & 4.217 & 31.12 & 40.22 & 34.27 \\
\hline
\end{tabular}

$\mathrm{SN}=$ Serial number; Means in a column followed by the same letter are not significantly different at $\mathrm{P}=0.05 ; \mathrm{LSD}=$ Least significant difference; Sed = Standard error of difference, MRN = Marketable root number per plot, TRN = Total root number per plot, MRW = Marketable root weight per plot (kg/plot), TRW = Total root weight per lot $(\mathrm{kg} / \mathrm{plot}), \mathrm{IRN}=\mathrm{Infested}$ root number per plot, IRW = Infested root weight per plot (kg/plot), WDS = Weevil damage score.

Table 4. Sweetpotato genotypes selected for weevil resistance and desirable yield and yield-related traits.

\begin{tabular}{llll}
\hline No. & Name & Weevil resistance & Agronomic or market traits \\
\hline 1 & Magunhwa & Resistant & Higher marketable root weight and total root weight per plot \\
2 & Chuchu ya Nesi & Resistant & $\begin{array}{l}\text { Higher total root number and lower infested root weight percent } \\
\text { per plot } \\
\text { Higher marketable root number per plot }\end{array}$ \\
3 & Rugomoka & Moderately resistant & $\begin{array}{l}\text { Higher marketable root number and root weight per plot } \\
\text { Higher marketable root number per plot }\end{array}$ \\
\hline
\end{tabular}

Table 5. Pearson correlation coefficients showing the association between root number and root weight with weevil damage and damage score.

\begin{tabular}{|c|c|c|c|c|c|c|c|}
\hline Traits & MRN & TRN & MRW & TRW & IRN & IRW & WDS \\
\hline MRN & & $0.73 * *$ & $0.76^{* *}$ & $0.77^{* *}$ & $0.37^{*}$ & $0.38^{*}$ & 0.20 \\
\hline TRN & & & $0.42 *$ & $0.54^{*}$ & $0.38^{*}$ & 0.25 & 0.02 \\
\hline MRW & & & & $0.96 * *$ & 0.22 & $0.45^{*}$ & 0.10 \\
\hline TRW & & & & & 0.26 & $0.45^{*}$ & 0.12 \\
\hline IRN & & & & & & $0.84 * *$ & $0.79 * *$ \\
\hline IRW & & & & & & & $0.72 * *$ \\
\hline WDS & & & & & & & \\
\hline
\end{tabular}

** = Significant correlation at $\mathrm{P}<0.01$ probability level; ${ }^{*}=$ Significant correlation at $\mathrm{P}<0.05$ probability level, MRN $=$ Marketable root number per plot, TRN $=$ Total root number per plot, $\mathrm{MRW}=$ Marketable root weight per plot $(\mathrm{kg} / \mathrm{plot}), \mathrm{TRW}=$ Total root weight per lot $(\mathrm{kg} / \mathrm{plot}), \mathrm{IRN}=$ Infested root number per plot, IRW $=$ Infested root weight per plot $(\mathrm{kg} / \mathrm{plot}), \mathrm{WDS}=\mathrm{Weevil}$ damage score.

Table 6. Principal component (PC) analysis showing the latent roots, \% variation and vector loadings of the first three PCs based on 7 variables used in the study.

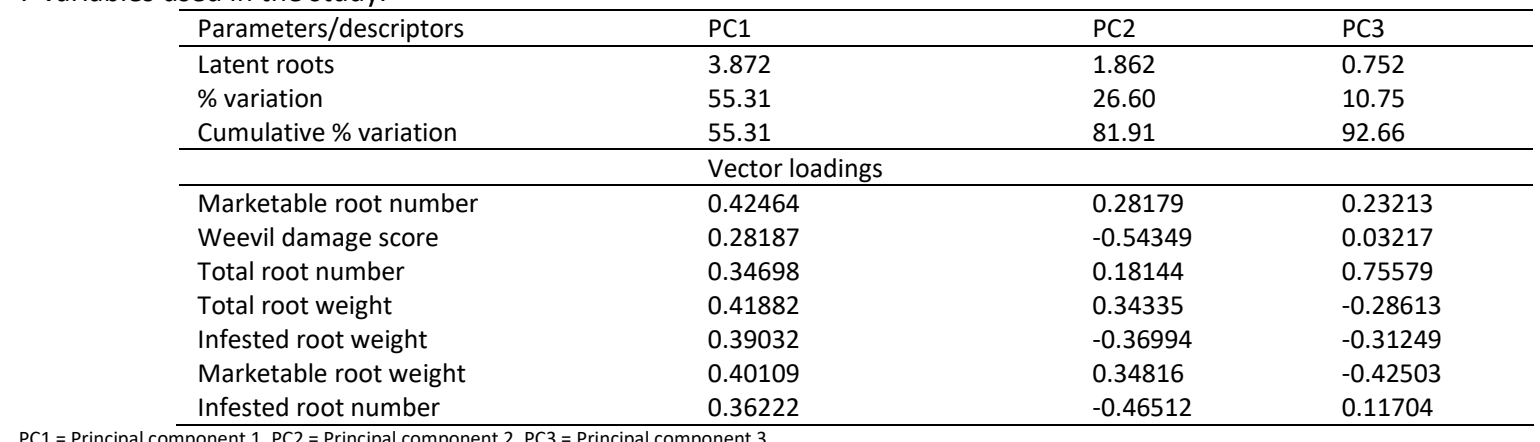

PC1 = Principal component 1, PC2 = Principal component 2, PC3 = Principal component 3.

Anyanga et al., 2013). In the studies conducted in Uganda, some weevil resistant sweetpotato varieties had high levels of esters of hydroxylcinnamic acid in root latex (Stevenson et study was 0.54 (Kagimbo et al., 2017). This suggested differential response of sweetpotato genotypes to weevil damage useful for future breeding programs.

The present study identified weevil resistant genotypes which included Kibandule, Mulumba, Utitiri, 3-CIP, Madebe, Magunhwa, 5-CIP, Kafu and Chuchu ya nesi. The genotypes Nyamvuva, Sengi, 22-CIP, Rugomoka, Tumauma, Ejumla, Carot C, New Kawogo 2, Haraka and 4-CIP were categorized as moderately weevil resistant collections (Table 3 ). The selected genotypes with resistance and moderate resistance reactions are ideal sweetpotao parents for weevil resistance breeding programs. Genotypes: Magunhwa, Chuchu ya Nesi, Rugomoka, Tumauma and New Kawogo were also identified al., 2009) and esters of caffeic and coumaric acid in epidermal and root surface (Anyanga et al., 2013). The genetic divergence of sweet potato genotypes used in this to be promising parents having both weevil resistance and desired yield and yield component traits.

\section{Association of yield and yield components with weevil infestation and damage}

The present study found a significant but weak correlation between TRN and IRN (Table 5). In addition, there were significant moderate correlations between MRW and TRW with IRW (Table 6). This association is supported by the results of PCA, which indicated that great variations among sweet potato genotypes was markedly contributed by marketable root number, total root number and marketable root weight (Table 6). As expected, these variables were 
positively correlated with weevil damage score. This suggests that sweetpotato varieties with numerous and bigger sized roots get more weevil infestations than those with few and small sized roots. Stathers et al. (2003) who indicated that sweetpotato genotypes, which produced higher numbers and thicker sized roots per plot, had higher, field weevil infestation and damage reported the same observations. Genotypes with thicker sized roots and high number of roots per plot tend to crack the soil creating access to storage roots by weevils resulting in heavy weevil infestation and damage (Skoglund and Smit, 1994; Stathers et al., 2003).

The significant correlation of both IRN and IRW with WDS revealed by this study indicates that high IRN reflects the level of susceptibility of a genotype to weevils. The main challenge encountered when assessing WDS is the occurrence of some sweetpotato genotypes expressing low levels of IRN but with high WDS. If both IRN and WDS are high (positively correlated) then it implies a high susceptibility to weevils. Sweetpotato genotypes expressing high levels of IRN but low WDS and vice versa can be attributed to having resistance to weevils or escape mechanism from weevils.

\section{Materials and methods}

\section{Description of the study sites}

This study was conducted under field experiments established at two sites. The first site was situated at Tumbi Agricultural Research Institute (ARI-Tumbi) located at $5^{\circ} 4^{\prime} 11^{\prime \prime} \mathrm{S}, 32^{\circ} 40^{\prime} 1^{\prime \prime} \mathrm{E}$ and the second site was at Ndorobo village located at $5^{\circ} 5^{\prime} 12^{\prime \prime} \mathrm{S}, 32^{\circ} 1^{\prime} 26^{\prime \prime} \mathrm{E}$ both in western Tanzania. ARI-Tumbi is characterized by a unimodal rainfall pattern receiving a mean annual rainfall of $920 \mathrm{~mm}$. The main rainfall season is between November and April. The climate is generally dry and warm with a mean daily temperature of $23^{\circ} \mathrm{C}$. These conditions favour sweetpotato weevil activity (Muyinza et al., 2012). Ndorobo village has a unimodal rainfall pattern receiving a mean annual rainfall of $960 \mathrm{~mm}$. It is generally dry and warm with a mean daily temperature of $22^{\circ} \mathrm{C}$. The two sites were identified as hotspots for sweetpotato weevils in western Tanzania. The soil physio-chemical characteristics of both sites selected for the study are presented in Table 1 whereas the rainfall distribution of the sites during the study season is presented in Figure 1.

\section{Germplasm and trial establishment}

The list of sweetpotato genotypes used in this study are presented in Supplementary Table 1. A collection of 96 sweetpotato genotypes were used in this study. Among the 96 genotypes, 57 were local varieties or landraces collected from western Tanzania; 19 were collected from Lake Zone and 20 genotypes were introductions from International Potato Centre (CIP)-Lima/Peru. The CIP collections were originally obtained from India. The genotype Simama was used as a susceptible check, while genotype New Kawogo was used as a resistant check (Muyinza et al., 2012). Genotypes were screened for weevil resistance under natural infestation at the selected hotspot areas. Trials were established in late January 2015 and harvested in late June
2015 to ensure high level of weevil infestation for selection. In the study areas, weevil infestation increases during late May coinciding with the dry spell.

Experiments were conducted using a $12 \times 8$ lattice design with three replications at each site. The spacing used was $1 \mathrm{~m}$ inter-row and $0.3 \mathrm{~m}$ intra-row. The plot size was $3 \mathrm{~m} \times 2.1$ $\mathrm{m}$ consisting of 3 ridges each with 7 plants making a total of 21 plants per plot. Fertilizers were applied using Nitrogen, Phosphorus and Potassium (15:9:20) at the rate of $233 \mathrm{~kg} / \mathrm{ha}$ a month after planting. Weeding was done three times at 30,70 and 110 days after planting.

\section{Data collection}

Six months after planting (MAP), the number of surviving plants in the middle row were recorded and the storage roots dug. The total weight of storage root per plot and the number of marketable and unmarketable storage roots per plot were recorded. Then the storage roots were separated into infested and non-infested roots and weighed separately. Infested roots were counted to get the infested root number (IRN) per plot. Weevil damage score (WDS) of roots was scored based on the proportion (percentage) of the root area damaged on the outer surface and inner parts of the root. WDS was assessed using a scale of 0 to 10 where $0=$ no damage, $1=1-10 \%$ of the root damaged, $2=11-20 \%$, $3=21-30 \%, 4=31-40 \%, 5=41-50 \%, 6=51-60 \%, 7=61$ $70 \%, 8=71-80 \%, 9=81-90 \%$ and $10=91-100 \%$.

\section{Data analysis}

Data collected were organised in excel and analysed using GENSTAT PROGRAM 14 ${ }^{\text {th }}$ edition (Payne et al., 2015). Marketable root number (MRN), total root number (TRN), marketable root weight (MRW), total root weight (TRW), IRN, infested root weight (IRW) and WDS were analysed using the restricted maximum likelihood (REML) procedure (Payne et al., 2015). Genotype was set as fixed effect, whereas season and genotype by season interaction, replication and block were treated as random effects. The model used was:

$Y i j k l=\mu+G i+L j+G L i j+R k+B I+\varepsilon i j k l$

Where: $\mu$ is the general mean, $G, L, G L, R$, and $B$ denote the effects of genotype, location, the interaction effects of genotype and location, replication, the incomplete block, in that order. $\varepsilon$ is the random term. Following significant tests, treatment means were separated using the least significant difference (LSD) procedure at $\mathrm{p} \leq 0.05$. The relationship between traits were assessed by computing the Pearson's correlation coefficients using SPSS 24 program (SPSS, 2009).

\section{Conclusion}

This study successfully identified weevil resistant genotypes which included: Kibandule, Malulumba, Utitiri, 3-CIP, Madebe, Magunhwa, 5-CIP, Kafu and Chuchu ya nesi. The genotypes Nyamvuva, sengi, 22-CIP, Rugomoka, Tumauma, Ejumla, Carot C, New Kawogo 2, Haraka and 4-CIP expressed moderate resistance to weevils. These genotypes were selected as the best parents to be used in breeding of sweetpotato varieties with enhanced weevil resistance. Furthermore, this study identified genotypes Magunhwa, Chuchu ya Nesi, Rugomoka, Tumauma and New Kawogo 
with weevil resistance and desired yield and yield-related traits such as high marketable root number, increased total root number per plot, high marketable root weight per plot and low infested root weight per plot. The study demonstrated that weevil infestation on storage roots was associated with sweetpotato root number per plot and large sized storage root. The study also indicated that the level of weevil infestation was associated with the level of damage on roots. The selected genotypes are recommended for weevil resistance breeding programs of sweetpotato in western Tanzania or similar agro-ecologies.

\section{Acknowledgments}

Appreciation to the Alliance for a Green Revolution in Africa (AGRA) for funding this study through the African Centre for Crop Improvement (ACCI).

\section{References}

Acquaah G (2012) Principles of plant genetics and breeding 2nd ed. Wiley-Blackwell, Oxford USA.

Anyanga MO, Muyinza H, Talwana H, Hall DR, Farman DI, Ssemakula GN, Mwanga ROM, Stevenson PC (2013) Resistance to the Weevils Cylas puncticollis and Cylas brunneus conferred by sweetpotato root surface compounds. J Agric Food Chem. 61:8141-8147.

Burri BJ (2011) Evaluating sweet potato as an intervention food to prevent vitamin A deficiency. Compr Rev Food Sci Food Saf. 10:118-130.

Clark CA, Davis JA, Abad JA, Cuellar WJ, Fuentes S, Kreuze JF, Gibson RW, Mukasa SB, Tugume AK, Tairo FD (2012) Sweetpotato viruses: 15 years of progress on understanding and managing complex diseases. Plant Dis. 96: 168-185.

Elameen A, Fjellheim S, Larsen A, Rognli OA, Sundheim L, Msolla S, Masumba E, Mtunda K, Klemsdal SS (2008) Analysis of genetic diversity in a sweet potato (Ipomoea batatas L.) germplasm collection from Tanzania as revealed by AFLP. Genet Resour Crop Evol. 55: 397-408.

Engoru P, Mugisha J, Bashaasha B (2005) Tuber utilization options among sweet potato producers in eastern Uganda. Afr Crop Sci J. 7:715-719.

FAOSTAT (2014) Food and Agriculture Organisation of the United Nations : Crop production data. FAOSTAT Division, Rome.

Fuglie KO (2007) Priorities for sweetpotato research in developing countries: Results of a survey. Hort Sci. 42:12001206.

Kagimbo FM, Shimelis H, Sibiya J (2017) Diversity assessment of sweetpotato germplasm collections for yield and yield-related traits in western Tanzania. Acta Agric Scand., Sect B:1-9. doi:10.1080/09064710.2017.1372516.

Kapinga RE, Ewell PT, Jeremiah S, Kileo R (1995) Sweetpotato in Tanzanian farming and food systems: implications for research. International Potato Center, Tanzanian Ministry of Agriculture, Nairobi and Dar-Es-Salaam.

Kulembeka H, Rugutu C, Kanju E, Chirimi B, Rwiza E, Amour R (2005) The agronomic performance and acceptability of orange fleshed sweetpotato varieties in the lake zone of Tanzania. Afr Crop Sci J. 12:229-240.

Lebot V (2009) Tropical root and tuber crops : cassava, sweet potato, yams and aroids. $\mathrm{CABI}$, Wallingford

Lebot V (2009) Sweetpotato. In: Bradshaw, J.E., editor, Root and tuber crops: Handbook of plant breeding. Springer Sciences and Business Media, New York, NY 10013, USA. p. 97-125.
Masumba E, Kulembeka H, Tollano S, Yongolo M (2005) Participatory evaluation of improved sweetpotato varieties in Eastern Tanzania. Afr Crop Sci J. 12:259-265.

Muyinza H, Talwana HL, Mwanga ROM, Stevenson PC (2012) Sweetpotato weevil (Cylas spp.) resistance in African sweetpotato germplasm. Int J Pest Manage. 58:73-81.

Mwanga $R$, Odongo $B$, Niringiye $C$, Kapinga $R$, Tumwegamire $S$, Abidin P, Carey E, Lemaga B, Nsumba J, Zhang D (2007) Sweetpotato selection releases: lessons learnt from Uganda. Afr Crop Sci J. 15: 11 - 23.

Schafleitner R, Tincopa LR, Palomino O, Rossel G, Robles RF, Alagon R, Rivera C, Quispe C, Rojas L, Pacheco JA (2010) A sweetpotato gene index established by de novo assembly of pyrosequencing and Sanger sequences and mining for genebased microsatellite markers. BMC Genomics 11: 604.

Nelles W (2009) Sweetpotato Education, Research and Capacity Development through a CIP-Orissa Learning Site. CTCRI, Bhubaneswar, Orissa, India.

Ngailo S, Shimelis H, Sibiya J, Mtunda K (2016a) Screening of Tanzanian sweet potato germplasm for yield and related traits and resistance to sweet potato virus disease. Acta Agric. Scand., Sect. B. 66:52-66.

Ngailo S, Shimelis H, Sibiya J, Mtunda K (2016b) Assessment of sweetpotato farming systems, production constraints and breeding priorities in eastern Tanzania. S Afr J Plant Soil 33:105-112.

Payne R, Welham S, Harding S (2015) A Guide to REML in Genstat. VSN International, 2 Amberside, Wood Lane, Hemel Hempstead, Hertfordshire HP2 4TP, UK.

Rukundo P, Shimelis H, Laing M, Gahakwa D (2013) Storage root formation, dry matter synthesis, accumulation and genetics in sweet potato. Aust J Crop Sci. 7:2054-2061.

Schafleitner R, Tincopa LR, Palomino O, Rossel G, Robles RF, Alagon R, Rivera C, Quispe C, Rojas L, Pacheco JA (2010) A sweetpotato gene index established by de novo assembly of pyrosequencing and Sanger sequences and mining for genebased microsatellite markers. BMC Genomics 11: 604 . doi.org/10.1186/1471-2164-11-604.

Skoglund LG, Smit NE (1994) Major diseases and pests of sweetpotato in Eastern Africa. International Potato Center (CIP).Nairobi, Kenya.

Smit NEJM, Downham MCA, Laboke PO, Hall DR, Odongo B (2001) Mass-trapping male Cylas spp. with sex pheromones: a potential IPM component in sweetpotato production in Uganda. Crop Prot. 20:643-651.

SPSS (2009) Statistical Package for Social Scientists. SPSS for Windows Release 18.0, Chicago, IL.

Stathers TE, Rees D, Kabi S, Mbilinyi L, Smit N, Kiozya H, Jeremiah S, Nyango A, Jeffries D (2003) Sweetpotato infestation by Cylas spp. in East Africa: I. Cultivar differences in field infestation and the role of plant factors. Int J Pest Manage. 49: 131-140.

Stevenson PC, Muyinza $H$, Hall DR, Porter DI, Farman $H$ Talwana, Mwanga ROM (2009) Chemical basis for resistance in sweetpotato Ipomoea batatas to the sweetpotato weevil Cylas puncticollis. Pure Appl Chem. 81:141-151.

Tairo F, Mneney E, Kullaya A (2008) Morphological and agronomical characterization of sweet potato [Ipomoea batatas (L.) Lam.] germplasm collection from Tanzania. Afr J Plant Sci. 2:77-85.

Talekar NS (1987) Feasibility of the use of resistant cultivar in sweetpotato weevil control. Int J Trop Insect Sci. 8:815-817. 